\title{
Relação entre alguns atributos físicos e a produção de grãos de soja e arroz de sequeiro em latossolos ${ }^{1}$
}

\author{
Relation among some physical attributes and grains production of soybean and rice in oxisols
}

\author{
Amauri Nelson Beutler ${ }^{1}$ José Frederico Centurion ${ }^{2}$ Cassiano Garcia Roque ${ }^{2}$
}

\section{RESUMO}

\begin{abstract}
Este trabalho objetivou avaliar atributos físicos e a correlação com a produção de grãos de soja e arroz de sequeiro em três níveis de compactação e dois conteúdos de água no solo (água retida na tensão de 0,05 e 0,01MPa), com três repetições. Foram utilizadas amostras de Latossolo Vermelho, textura média (LVd) e, Latossolo Vermelho, textura argilosa (LVef), coletadas na profundidade de $0-20 \mathrm{~cm} e$ compactadas em camadas de $3 \mathrm{~cm}$, em vasos de 9,82L. Foram avaliados a porosidade total, macro e microporosidade, densidade do solo, resistência à penetração e a produção de grãos de soja e arroz de sequeiro. Os atributos físicos apresentaram correlações altas e significativas entre si, nos dois solos e conteúdos de água. As maiores correlações dos atributos físicos com a produção de grãos de soja e arroz foram obtidas no LVd e no conteúdo de água retida na tensão de 0,05MPa, condição em que ocorreu maior redução da produção de grãos com o aumento da compactação. As correlações foram positivas da macroporosidade e porosidade total e negativas da microporosidade, densidade do solo e resistência à penetração com a produção de grãos de soja e arroz.
\end{abstract}

Palavras-chave: compactação do solo, conteúdo de água, Glycine max, Oryza sativa.

\section{ABSTRACT}

This work aimed to evaluate some physical attributes and their correlation with production of soybean (Glycine max) and rice (Oryza sativa) in three levels of soil compaction and two water contents (tension of 0.01 and $0.05 \mathrm{MPa}$ ), with three replications. Samples of a Haplustox and a Eutrustox were collected at $0-20 \mathrm{~cm}$ depth and compacted in layers of $3 \mathrm{~cm}$ in pots of $9.82 \mathrm{~L}$. The total porosity, macro and microporosity, bulk density, penetration resistance and soybean and rice grain production were evaluated. The physical attributes presented high and significant correlations among them for all soils na water contents. The greater correlations of physical attributes with soybean and rice grain production were obtained in Haplustox at water content at $0.05 \mathrm{MPa}$, condition where greater reduction of grains production with increase of compaction occurred. The correlations of macroporosity and total porosity with grain production of both crops were positive, while microporosity, bulk density and penetration resistance correlations were negative.

Key words: soil compaction, water content, Glycine max, Oryza sativa.

\section{INTRODUÇÃO}

As culturas de soja e arroz de sequeiro são cultivadas em extensas áreas agrícolas e em grande diversidade de solos, sendo que, a partir de 1980, ocorreu um aumento gradativo na utilização de sistemas de manejo com revolvimento mínimo do solo, denominado plantio direto. Esse sistema atualmente ocupa grandes áreas no sul e no cerrado brasileiro devido a vantagens de ordem técnica, econômica e ambiental. Pode-se citar a menor utilização de máquinas agrícolas para preparo do solo e conseqüente redução de custos, e redução nas perdas de solo, nutrientes e água, o que, por sua vez, reduz a poluição de mananciais de água, além de preservar a estrutura do solo. No entanto, pesquisadores têm verificado que o acentuado tráfego de máquinas e equipamentos pesados sobre o solo provoca compactação superficial do solo até a profundidade de 15 a 20cm (BEUTLER et al., 2001), fato que tem dificultado a expansão do sistema de plantio direto.

${ }^{1}$ Parte da tese de Doutorado do primeiro autor.

${ }^{2}$ Aluno de Doutorado, Universidade Estadual Paulista, Faculdade de Ciências Agrárias e Veterinárias (FCAV), Universidade do Estado de São Paulo (UNESP), Via de Acesso Rod. Paulo Donato Castellane, s/n, 14880-000, Jaboticabal, SP. Bolsista da Fapesp. amaurib@yahoo.com.br. Autor para correspondência.

${ }^{3}$ Professor Adjunto, FCAV, UNESP, Jaboticabal. Jfcentur@fcav.unesp.br 
Em solos compactados, o desenvolvimento das plantas é menor (VIEIRA \& MUZILLI, 1984; TORMENA et al., 1998; MEROTTO \& MUNDSTOCK, 1999; GUIMARÃES et al., 2002). Isto tem sido atribuído ao impedimento mecânico do solo e ao crescimento radical, que resulta em menor volume de solo explorado, menor absorção de água e nutrientes e, consequentemente menor produção das culturas. Por outro lado, ANDRADE et al. (1993) verificaram menor crescimento das plantas de girassol sob estresse físico provocado pela compactação do solo, o qual não foi influenciado pelo suprimento de água, oxigênio, nutrientes e disponibilidade de carboidratos.

Para avaliar a compactação do solo em relação à produtividade das culturas, são utilizados alguns atributos físicos. Segundo LETEY (1985), os atributos físicos são classificados como diretamente e indiretamente relacionados com o crescimento das plantas, sendo a água, o oxigênio, a temperatura e a resistência do solo à penetração os que têm relações diretas com o crescimento radical, e a densidade do solo, a porosidade, a infiltração de água, a agregação e a textura os de relações indiretas. Dentre estes, é importante a seleção e utilização de atributos físicos sensíveis ao manejo e relacionados com a produção, monitoramento da qualidade do solo, maximização da produção das culturas e manutenção da sustentabilidade dos sistemas agrícolas, minimizando a degradação dos solos e do meio ambiente.

A compactação é caracterizada pelo aumento da resistência do solo à penetração, da densidade do solo e da microporosidade, com redução da porosidade total e da macroporosidade (ALVARENGA et al., 1996; MEROTTO \& MUNDSTOCK, 1999; ROSOLEM et al., 1999; BEUTLER et al., 2002; STONE et al., 2002). Em geral, pequenas compactações são benéficas por aumentar a área de contato solo/raiz, proporcionando um adequado suprimento de água e nutrientes à parte aérea (KOOISTRA et al., 1992). Entretanto, o valor de resistência do solo à penetração de $2 \mathrm{MPa}$ é considerado como restritivo ao desenvolvimento das plantas (TORMENA et al., 1998).

Os solos apresentam comportamentos físicos distintos de acordo com a composição granulométrica, mineralogia (FERREIRA et al., 1999), teor de matéria orgânica e a umidade. Neste sentido, HAMBLIM (1985) afirmou que a resposta das culturas à compactação do solo é acentuadamente influenciada pela condição estrutural do solo. Ainda, DEXTER (1987) afirma que a compactação do solo é mais prejudicial em solo seco, sendo que, em condições de maior umidade pode haver crescimento radical em valores de resistência à penetração superiores a 4MPa.

Este estudo objetivou avaliar atributos físicos e as correlações com a produção de grãos de soja e arroz de sequeiro, em dois conteúdos de água no solo e em diferentes níveis de compactação de um Latossolo Vermelho, textura média (LVd) e um Latossolo Vermelho argiloso (LVef).

\section{MATERIAL E MÉTODOS}

O experimento foi conduzido em casa de vegetação do Departamento de Solos e Adubos da FCAV/UNESP de Jaboticabal (SP), em Latossolo Vermelho distrófico típico textura média A moderado caulinítico hipoférrico (LVd), e Latossolo Vermelho eutroférrico típico textura argilosa A moderado caulinítico-oxídico (LVef). A composição granulométrica foi determinada em amostras deformadas através da dispersão com $\mathrm{NaOH}(0,1$ mol $\left.\mathrm{L}^{-1}\right)$ e agitação lenta durante 16 horas, sendo o conteúdo de argila obtido pelo método da pipeta (DAY, 1965). A densidade de partículas foi determinada pelo método do picnômetro, segundo BLAKE \& HARTGE (1986a). Os solos LVd e LVef apresentaram, respectivamente, 271 e $517 \mathrm{~g} \mathrm{~kg}^{-1}$ de argila; 42 e $256 \mathrm{~g}$ $\mathrm{kg}^{-1}$ de silte; 687 e $227 \mathrm{~g} \mathrm{~kg}^{-1}$ de areia; e densidade de partículas de 2,82 e $2,98 \mathrm{Mg} \mathrm{m}^{-3}$.

As amostras dos solos foram coletadas na camada de $0-20 \mathrm{~cm}$ e passadas em peneira de $0,4 \mathrm{~cm}$. $\mathrm{O}$ solo utilizado para cada cultura foi adubado segundo RAIJ et al. (1996), cuja análise química seguiu a metodologia de RAIJ et al. (1987). A caracterização química no momento da colheita mostrou níveis médios de fósforo e potássio, altos de cálcio e magnésio (relação 2:1) e saturação de bases de $68 \%$, para soja e arroz, no LVd e LVef.

Após a realização da adubação, foram ajustados os conteúdos de água de 0,11 e $0,14 \mathrm{~kg} \mathrm{~kg}^{-1}$ no LVd e 0,24 e $0,27 \mathrm{~kg} \mathrm{~kg}^{-1}$ no LVef, correspondentes aos conteúdos de água retida nas tensões de $0,05 \mathrm{e}$ 0,01MPa, respectivamente. Estes conteúdos de água foram determinados previamente em câmaras de Richards em amostras indeformadas, segundo KLUTE (1986). Em seguida, o solo foi colocado em vaso cilíndrico de PVC com capacidade de 9,82L $(20 \mathrm{~cm}$ de altura e $25 \mathrm{~cm}$ de diâmetro), em camadas de $3 \mathrm{~cm}$. Cada camada foi compactada através da queda livre de um êmbolo de $7 \mathrm{~kg}$, da altura de $60 \mathrm{~cm}$, no centro geométrico de uma superfície de madeira com diâmetro ligeiramente inferior ao vaso, semelhante ao descrito por MORAES et al. (1991). Foram estabelecidos três níveis de resistência do solo à penetração. 
Em vasos com níveis de compactação e umidade equivalentes aos utilizados para o cultivo, foram coletadas amostras indeformadas, na profundidade de $2-5 \mathrm{~cm}$, com anéis volumétricos de $3 \mathrm{~cm}$ de altura e $4,8 \mathrm{~cm}$ de diâmetro para determinação da densidade do solo (Ds) segundo BLAKE \& HARTGE (1986b), da microporosidade (Mi), por secagem (tensão de 0,006 MPa), em câmaras de pressão de Richards com placa porosa (KLUTE, 1986), da porosidade total $(\mathrm{Pt})$ segundo DANIELSON \& SUTHERLAND (1986), e a macroporosidade (Ma) foi obtida por diferença entre a Pt e a Mi.

A resistência à penetração $(\mathrm{Rp})$ foi determinada com o penetrômetro de anel dinamométrico (Solotest 1.210.001), com ângulo de cone de $30^{\circ}$. As leituras foram realizadas quando a base do cone atingiu a profundidade de $3 \mathrm{~cm}$, e o valor de cada repetição foi obtido da média de quatro subdeterminações. Foram utilizados cones com área de 6,33; 3,8; 1,13 e 0,28 $\mathrm{cm}^{2}$, respectivamente, diminuindo a área do cone com o incremento da compactação do solo. $\mathrm{O}$ valor obtido no relógio de leitura foi transformado em kgf pela equação linear de calibração do aparelho, a seguir: $\mathrm{R}=1,0444+0,2998 x$; em que $R$ é a resistência à penetração em kgf, $x$ é a leitura no relógio. $\mathrm{O}$ valor obtido em kgf foi dividido pela área do cone e multiplicado pelo fator 0,098 para transformação em MPa.

Foram semeadas três sementes por cova (4 covas/vaso) de soja (Glycine $\max \boldsymbol{c v}$. EMBRAPA 48) e arroz (Oryza sativa cv. IAC 165), em 2001. Após sete dias, realizou-se o desbaste, deixando-se duas plantas de soja e quatro plantas de arroz por vaso. Nesta data, foram aplicados os tratamentos de umidade, visto que foi necessário aumentar o conteúdo de água no solo para possibilitar a germinação nos vasos mais secos e compactados. A umidade foi mantida constante, através de duas pesagens diárias dos vasos referentes a uma repetição e reposição de água através de tubo de PVC perfurado, instalado no centro geométrico do vaso, sendo feita a pesagem e rodízio de todos os vasos a cada 5 dias.

Foi avaliada a produção de grãos de soja e arroz por planta, sendo a umidade dos grãos ajustada a $0,12 \mathrm{~kg} \mathrm{~kg}^{-1}$ por $\mathrm{P}=\mathrm{Pi} *((100-\mathrm{Ui}) /(100-\mathrm{Uf}))$, em que $\mathrm{P}$ é a produção final $\left(\mathrm{g}\right.$ planta-1) $\mathrm{com} 0,12 \mathrm{~kg} \mathrm{~kg}^{-1} \mathrm{de}$ umidade nos grãos, Pi é a produção inicial $\left(\mathrm{g} \mathrm{planta}^{-1}\right)$, Ui é a umidade inicial $\left(\mathrm{kg} \mathrm{kg}^{-1}\right)$ e Uf é a umidade final $\left(0,12 \mathrm{~kg} \mathrm{~kg}^{-1}\right)$.

Os tratamentos consistiram de três níveis de compactação e dois conteúdos de água, constituindo um experimento inteiramente casualizado, em esquema fatorial $3 \times 2$, com três repetições. Foram realizadas correlações entre os atributos físicos e destes com a produção de grãos de soja e arroz de sequeiro. As análises foram processadas por meio do Statistical Analysis System (SAS, 1995).

\section{RESULTADOS E DISCUSSÃO}

Os valores dos atributos físicos e da produção de grãos de soja e arroz no LVd e LVef, nos dois conteúdos de água são apresentados na tabela 1 . Verifica-se que o menor nível de compactação foi próximo a $\mathrm{Rp}$ de $2 \mathrm{MPa}$, adotado como limitante ao crescimento radical e da parte aérea das plantas (TORMENA et al., 1998). Assim, as correlações dos atributos físicos com a produção das culturas foram realizadas na faixa de compactação prejudicial ao desenvolvimento das plantas. A Pt e a Ma diminuíram e a Mi aumentou com o incremento da Rp e da Ds (Tabela 1). Resultados semelhantes foram encontrados por ALVARENGA et al. (1996), MEROTTO \& MUNDSTOCK (1999), BEUTLER et al. (2002), STONE et al. (2002). Entretanto, verifica-se que o incremento da Mi foi de 4,2 e 5,5\%, valor inferior relativo ao decréscimo da Pt de $-7,1$ e -7,3\% e da $\mathrm{Ma}-31,4$ e $53,0 \%$ no LVd no conteúdo de água retida a $0,05 \mathrm{MPa}$, fato que se repete na outra condição de umidade e solo. Neste sentido, MEROTTO \& MUNDSTOCK (1999) e STONE et al. (2002) verificaram incremento não significativo da Mi com o aumento da Ds; e STONE et al. (2002) observaram que o aumento da Ds se refletiu na redução da Pt e em maior proporção da Ma.

É importante mencionar que foram encontradas altas correlações significativas a $1 \%$ de probabilidade entre os atributos físicos, e com valores acima de 0,92 para a Ds, Ma, Mi e Pt (Tabela 2). VIEIRA \& MUZILLI (1984), estudando atributos físicos em sistemas de plantio direto e convencional e rotação de culturas com milho, soja e trigo, verificaram, após quatro anos, correlações de 0,97, 0,87 e 0,99 para a Ma, Mi e Pt com a Ds na profundidade de $10 \mathrm{~cm}$.

As altas correlações entre a Ds e Pt, Ds e $\mathrm{Ma}$ (negativas) e Ds e Mi (positivas) ocorrem, segundo STONE et al. (2002), devido à aproximação das partículas com o incremento da pressão mecânica exercida sobre o solo, reduzindo a proporção dos poros de maior diâmetro e incrementando ligeiramente os de menor diâmetro.

Foram obtidas equações quadráticas com altos coeficientes de determinação entre a Rp e a Ds, nos dois solos e conteúdos de água (Tabela 2). STONE et al. (2002) encontraram equação quadrática com coeficiente de determinação de 0,96 entre a Rp e a Ds em Latossolo Vermelho argiloso; e ROSOLEM 
Tabela 1 - Porosidade total $(\mathrm{Pt})$, macro $(\mathrm{Ma})$ e microporosidade (Mi), densidade do solo (Ds) e resistência do solo à penetração (Rp) e produção de grãos de soja e arroz/planta, em níveis crescentes de compactação, no conteúdo de água retida nas tensôes de 0,05 e 0,01MPa no Latossolo Vermelho textura média (LVd) e no Latossolo Vermelho argiloso (LVef).

\begin{tabular}{|c|c|c|c|c|c|c|c|c|c|c|c|c|c|}
\hline \multicolumn{7}{|c|}{$0,05 \mathrm{MPa}$} & \multicolumn{7}{|c|}{$0,01 \mathrm{MPa}$} \\
\hline & \multirow{2}{*}{$\frac{\mathrm{Ma}}{\mathrm{m}^{3} \mathrm{~m}^{-3}---}$} & \multirow[t]{2}{*}{$\mathrm{Mi}$} & \multirow{2}{*}{$\frac{\mathrm{Ds}}{\mathrm{Mg} \mathrm{m}^{-3}}$} & \multirow{2}{*}{$\frac{\mathrm{Rp}}{\mathrm{MPa}}$} & \multicolumn{2}{|c|}{ Produção (g) } & \multirow[t]{2}{*}{$\mathrm{Pt}$} & \multirow{2}{*}{$\frac{\mathrm{Ma}}{\mathrm{m}^{3} \mathrm{~m}^{-3}-}$} & \multirow{2}{*}{ Mi } & \multirow{2}{*}{$\frac{\mathrm{Ds}}{\mathrm{Mg} \mathrm{m}^{-3}}$} & \multirow{2}{*}{$\frac{\mathrm{Rp}}{\mathrm{MPa}}$} & \multicolumn{2}{|c|}{ Produção (g) } \\
\hline & & & & & Soja & Arroz & & & & & & Soja & Arroz \\
\hline \multicolumn{14}{|c|}{ LVd } \\
\hline 0,391 & 0,155 & 0,236 & 1,46 & 2,04 & 6,29 & 1,70 & 0,386 & 0,141 & 0,245 & 1,43 & 1,79 & 13,61 & 6,16 \\
\hline 0,367 & 0,118 & 0,249 & 1,51 & 2,75 & 5,73 & 1,06 & 0,372 & 0,124 & 0,248 & 1,54 & 2,32 & 13,48 & 5,27 \\
\hline 0,365 & 0,118 & 0,246 & 1,54 & 4,18 & 3,23 & 0,77 & 0,330 & 0,064 & 0,266 & 1,59 & 3,58 & 11,29 & 5,09 \\
\hline \multicolumn{14}{|c|}{ LVef } \\
\hline 0,500 & 0,153 & 0,347 & 1,20 & 2,52 & 12,06 & 1,09 & 0,484 & 0,126 & 0,358 & 1,24 & 1,78 & 18,71 & 4,45 \\
\hline 0,484 & 0,132 & 0,352 & 1,24 & 3,83 & 11,22 & 0,99 & 0,466 & 0,097 & 0,369 & 1,28 & 2,54 & 17,46 & 3,95 \\
\hline 0,466 & 0,100 & 0,366 & 1,30 & 4,32 & 10,41 & 0,89 & 0,442 & 0,063 & 0,379 & 1,39 & 4,03 & 17,00 & 2,95 \\
\hline
\end{tabular}

et al. (1999) verificaram aumento quadrático e logarítmico da Rp em função da Ds com coeficientes de determinação acima de 0,98 , para solos com menos e mais de $400 \mathrm{~g} \mathrm{~kg}^{-1}$ de argila, respectivamente. Estes autores verificaram ainda que, próximo a Ds máxima, o valor de Rp foi superior e aumentou em maior proporção com a Ds em solos mais argilosos. Isto demonstra que a $\mathrm{Rp}$ é extremamente dependente da Ds.

A Pt e a Ma apresentaram correlações significativas e positivas, e a $\mathrm{Mi}$, a Ds e a $\mathrm{Rp}$ correlações negativas com a produção de grãos de soja e arroz no LVd, nos dois conteúdos de água (Tabela 3). Já no LVef, as correlações foram significativas apenas para o arroz no conteúdo de água retida na tensão de 0,01 $\mathrm{MPa}$, apesar de apresentarem correlações significativas a $15,0 \%$ de probabilidade nas demais condições. Este resultado demonstra que no LVd textura média a produção de grãos de soja e arroz foi mais influenciada pelos atributos físicos. Neste contexto, HAMBLIM (1985) menciona que a resposta das plantas a compactação do solo é

Tabela 2 - Correlações de Pearson entre a porosidade total (Pt), macro (Ma) e microporosidade (Mi), densidade do solo (Ds) e resistência do solo à penetração $(\mathrm{Rp})$, e, regressões entre a Rp e a Ds, no conteúdo de água retida nas tensões de 0,05 e 0,01MPa no Latossolo Vermelho textura média (LVd) e no Latossolo Vermelho argiloso (LVef).

\begin{tabular}{|c|c|c|c|c|c|c|c|c|}
\hline & \multicolumn{8}{|c|}{ Correlação de Pearson (r) } \\
\hline & \multicolumn{4}{|c|}{$0,05 \mathrm{MPa}$} & \multicolumn{4}{|c|}{$0,01 \mathrm{MPa}$} \\
\hline & $\mathrm{Ma}$ & Mi & Ds & $\mathrm{Rp}$ & $\mathrm{Ma}$ & Mi & Ds & $\mathrm{Rp}$ \\
\hline & \multicolumn{8}{|c|}{ LVd } \\
\hline $\mathrm{Pt}$ & 0,99 & $-0,99$ & $-0,96$ & $-0,80$ & 0,99 & $-0,99$ & $-0,92$ & $-0,99$ \\
\hline $\mathrm{Ma}$ & & $-0,99$ & $-0,96$ & $-0,82$ & & $-0,99$ & $-0,92$ & $-0,99$ \\
\hline \multirow[t]{2}{*}{$\mathrm{Mi}$} & & & 0,97 & 0,85 & & & 0,92 & 0,99 \\
\hline & & & & & & & & \\
\hline $\mathrm{Pt}$ & 0,99 & $-0,93$ & $-0,99$ & $-0,96$ & 0,99 & $-0,99$ & $-0,99$ & $-0,99$ \\
\hline $\mathrm{Ma}$ & & $-0,98$ & $-0,99$ & $-0,90$ & & $-0,99$ & $-0,99$ & $-0,99$ \\
\hline $\mathrm{Mi}$ & & & 0,95 & 0,80 & & & 0,99 & 0,98 \\
\hline Solo - Tensão & & & \multicolumn{3}{|c|}{ Equação de regressão } & \multicolumn{3}{|c|}{$\mathrm{R}^{2}$} \\
\hline LVd $(0,05)$ & & & \multicolumn{3}{|c|}{$\mathrm{Rp}=903,6-1228,2 \mathrm{Ds}+4187,3 \mathrm{Ds}^{2}$} & \multicolumn{3}{|c|}{0,99} \\
\hline$(0,01)$ & & & \multicolumn{3}{|c|}{$\mathrm{Rp}=208,9-285,2 \mathrm{Ds}+98,1 \mathrm{Ds}^{2}$} & \multicolumn{3}{|c|}{0,99} \\
\hline LVef $(0,05)$ & & & \multicolumn{3}{|c|}{$\mathrm{Rp}=-402,6+632,6 \mathrm{Ds}-245,8 \mathrm{Ds}^{2}$} & \multicolumn{3}{|c|}{0,99} \\
\hline$(0,01)$ & & & \multicolumn{3}{|c|}{$\mathrm{Rp}=-79,5+110,6 \mathrm{Ds}-36,4 \mathrm{Ds}^{2}$} & \multicolumn{3}{|c|}{0,99} \\
\hline
\end{tabular}

Todas as correlações foram significativas em $1 \%$ de probabilidade. 
Tabela 3 - Correlações de Pearson entre a porosidade total (Pt), macro (Ma) e microporosidade (Mi), densidade do solo (Ds) e resistência do solo à penetração $(\mathrm{Rp})$ com a produção de grãos de soja e arroz/planta, no conteúdo de água retida nas tensões de 0,05 e $0,01 \mathrm{MPa}$, no Latossolo Vermelho textura média (LVd) e no Latossolo Vermelho argiloso (LVef).

\begin{tabular}{|c|c|c|c|c|}
\hline \multirow{3}{*}{ Atributos físicos } & \multicolumn{4}{|c|}{ Correlação com a produção de grãos/planta } \\
\hline & \multicolumn{2}{|c|}{$0,05 \mathrm{MPa}$} & \multicolumn{2}{|c|}{$0,01 \mathrm{MPa}$} \\
\hline & $\mathrm{r}$ & Probabilidade & $\mathrm{r}$ & Probabilidade \\
\hline \multicolumn{5}{|c|}{ SOJA } \\
\hline \multicolumn{5}{|c|}{ LVd } \\
\hline Pt & $0,91 * *$ & 0,0017 & $0,67^{*}$ & 0,0499 \\
\hline Ma & $0,91 * *$ & 0,0016 & $0,67 *$ & 0,0474 \\
\hline $\mathrm{Mi}$ & $-0,92 * *$ & 0,0012 & $-0,68 *$ & 0,0423 \\
\hline Ds & $-0,93 * *$ & 0,0009 & $-0,82^{* *}$ & 0,0073 \\
\hline $\mathrm{Rp}$ & $-0,85^{* *}$ & 0,0027 & $-0,70 *$ & 0,0375 \\
\hline \multicolumn{5}{|c|}{ LVef } \\
\hline $\mathrm{Pt}$ & $0,58^{\mathrm{ns}}$ & 0,0980 & $0,65^{\mathrm{ns}}$ & 0,0588 \\
\hline $\mathrm{Ma}$ & $0,60^{\mathrm{ns}}$ & 0,0880 & $0,65^{\mathrm{ns}}$ & 0,0601 \\
\hline $\mathrm{Mi}$ & $-0,60^{\mathrm{ns}}$ & 0,0910 & $-0,64^{\mathrm{ns}}$ & 0,0629 \\
\hline Ds & $-0,59^{\text {ns }}$ & 0,0944 & $-0,63^{\text {ns }}$ & 0,0666 \\
\hline $\mathrm{Rp}$ & $-0,53^{\mathrm{ns}}$ & 0,1466 & $-0,65^{\mathrm{ns}}$ & 0,0566 \\
\hline \multicolumn{5}{|c|}{ ARROZ } \\
\hline \multicolumn{5}{|c|}{ LVd } \\
\hline $\mathrm{Pt}$ & $0,68 *$ & 0,0429 & $0,75^{*}$ & 0,0203 \\
\hline $\mathrm{Ma}$ & $0,70^{*}$ & 0,0350 & $0,75^{*}$ & 0,0207 \\
\hline $\mathrm{Mi}$ & $-0,73^{*}$ & 0,0245 & $-0,74 *$ & 0,0217 \\
\hline Ds & $-0,86^{* *}$ & 0,0031 & $-0,63^{\mathrm{ns}}$ & 0,0688 \\
\hline $\mathrm{Rp}$ & $-0,97 * *$ & 0,0001 & $-0,74 *$ & 0,0229 \\
\hline \multicolumn{5}{|c|}{ LVef } \\
\hline $\mathrm{Pt}$ & $0,60^{\mathrm{ns}}$ & 0,0878 & $0,74 *$ & 0,0215 \\
\hline $\mathrm{Ma}$ & $0,59^{\mathrm{ns}}$ & 0,0952 & $0,75^{*}$ & 0,0196 \\
\hline $\mathrm{Mi}$ & $-0,55^{\mathrm{ns}}$ & 0,1224 & $-0,76^{*}$ & 0,0170 \\
\hline Ds & $-0,60^{\text {ns }}$ & 0,0887 & $-0,77^{*}$ & 0,0150 \\
\hline $\mathrm{Rp}$ & $-0,58^{\mathrm{ns}}$ & 0,1005 & $-0,71^{*}$ & 0,0314 \\
\hline
\end{tabular}

**- significativo a $1 \%$; *- significativo a $5 \% ;{ }^{\text {ns }}$ - não significativo.

dependente da estrutura do solo, principalmente da porosidade; e MORAES et al. (1991) observaram que o mesmo nível de compactação foi mais prejudicial ao desenvolvimento de soja em Latossolo Roxo, comparado à Terra Roxa Estruturada mais argilosa.

No conteúdo de água retida na tensão de 0,05MPa, no LVd, as correlações entre os atributos físicos e a produção de grãos foram significativas e superiores para soja, comparado a 0,01MPa. Já para o arroz as correlação foram superiores no LVd apenas para a Ds e Rp, comparada à tensão de $0,01 \mathrm{MPa}$. Ainda, a produção de grãos de soja no LVd (Tabela 1) foi reduzida em 48,7 e 17,0\% quando a Rp passou de 2,04 a 4,18MPa e de 1,79 a 3,58MPa, no conteúdo de água retida nas tensões de 0,05 e $0,01 \mathrm{MPa}$, respectivamente. A maior relação dos atributos físicos com a produção de grãos em menores conteúdos de água no solo é mencionada por DEXTER (1987), que verificou que em baixos conteúdos de água no solo, valores de Rp de $1 \mathrm{MPa}$ podem restringir o crescimento radical, e que em solos mais úmidos, pode haver crescimento radical em valores de Rp superiores a 4MPa.

As correlações significativas e positivas da Ma com a produção de grãos de soja e arroz no LVd ocorrem, segundo TIMLIM et al. (1994), devido ao fato do incremento da densidade de macroporos aumentar o crescimento das raízes e a quantidade de solo explorado pelas raízes e, conseqüentemente, proporcionando maior absorção de água e nutrientes, resultando em maior produção de grãos. Entretanto, para o LVef a correlação foi menor $(r=0,59$ a 0,75$)$, devido a maior Pt do solo. Neste sentido, KOPI \& 
DOUGLAS (1991) verificaram que uma grande área de contato solo/raiz, espaço poroso suficiente para o movimento de água e gases e baixa Rp é a estrutura ideal ao desenvolvimento das plantas; e KOOISTRA et al. (1992) observaram efeitos benéficos com uma pequena compactação em solos muito porosos. MORAES et al. (1998) encontraram correlação de 0,68 , significativa a $5 \%$ de probabilidade, entre a Ma e a produção de matéria seca de feijão em Latossolo Roxo argiloso e Terra Roxa Estruturada, com Ma acima de $0,18 \mathrm{~m}^{3} \mathrm{~m}^{-3}$, no maior nível de compactação.

As maiores correlações dos atributos físicos com a produção de grãos de soja e arroz no LVd mostram a importância da mineralogia caulinítica, pois promove a formação da estrutura em blocos com aspecto maciça coesa "in situ", com menor porosidade em relação ao LVef, cuja mineralogia oxídica promove a formação de microagregados com menos de $1 \mathrm{~mm}$ de diâmetro, formando uma estrutura granular com aspecto maciça porosa "in situ”, com maior proporção de poros de maior diâmetro (FERREIRA et al., 1999). A estrutura granular e a melhor distribuição do sistema radical das culturas no LVef na tensão de 0,05 $\mathrm{MPa}$ foram observadas visualmente, fatores estes responsáveis pela menor redução da produção com o incremento da resistência à penetração 13,7 e 18,3\% comparado ao LVd com 48,7 e 54,7\%, para soja e arroz, respectivamente.

As correlações entre a Ma, Mi, Pt, Ds e Rp com a produção de grãos, na tensão de $0,05 \mathrm{MPa}$ no $\mathrm{LVd}$, foram próximas para a soja e, a Rp seguida da Ds apresentaram as maiores correlações com a produção de arroz nesta condição (Tabela 3). Na tensão de 0,01MPa no LVd a Rp apresentou correlações próximas com a produção de arroz Ma, Mi e Pt. Já TAYLOR \& GARDNER (1963) encontraram maior correlação da Rp $(r=-0,96)$, comparado a Ds $(r=-0,59)$ com o crescimento radical de algodão. Além disso, a Rp é um atributo físico diretamente relacionado com a resposta fisiológica e o crescimento das plantas em solos compactados (LETEY, 1985), e está sendo utilizado como atributo para definir níveis de compactação, a partir do qual devem ser tomadas medidas mecânicas ou biológicas de descompactação, para permitir o adequado desenvolvimento das plantas e maximizar a produção (TORMENA et al., 1998).

\section{CONCLUSÕES}

A resistência à penetração, a densidade do solo, a porosidade total, a macro e microporosidade apresentaram correlações altas entre si, nos dois solos e conteúdos de água. $\mathrm{O}$ aumento da resistência do solo à penetração, da densidade e microporosidade do solo resultou em decréscimo da produção de grãos de soja e arroz de sequeiro. Os atributos físicos tiveram maiores relações com a produção de grãos no menor conteúdo de água no solo e no Latossolo Vermelho de textura média.

\section{REFERÊNCIAS BIBLIOGRÁFICAS}

ALVARENGA, R.C. et al.. Crescimento de raízes de leguminosas em camadas de solo compactadas artificialmente. Revista Brasileira de Ciência do Solo, Campinas, v.20, p.319-326, 1996.

BEUTLER, A.N. et al. Utilização dos penetrômetros de impacto e de anel dinamométrico em Latossolos. Engenharia Agrícola, Jaboticabal, v.22, p.191-199, 2002.

BEUTLER, A.N. et al. Resistência à penetração e permeabilidade de latossolo Vermelho distrófico típico sob sistemas de manejo na região dos cerrados. Revista Brasileira de Ciência do Solo, Viçosa, v.25, p.167-177, 2001.

BLAKE, G.R.; HARTGE, K.H. Particle density. In: KLUTE, A. Methods of soil analysis. 2.ed. Madison : American Society of Agronomy, 1986a. V.1, p.377-382.

BLAKE, G.R.; HARTGE, K.H. Bulk density. In: KLUTE, A. Methods of soil analysis. 2.ed. Madison : American Society of Agronomy, 1986b. V.1, p.363-375.

DANIELSON, R.E.; SUTHERLAND, P.L. Porosity. In: KLUTE, A. Methods of soil analysis. 2.ed. Madison : American Society of Agronomy, 1986. Part 1, p.443461 .

DAY, P.R. Particle fractionation and particle size analyses. In: ___ Methods of soil analysis. Madison : American Society of Agronomy, 1965. p.545-566.

DEXTER, A.R. Mechanics of root growth. Plant and Soil, Dorbrecht, v.98, p.303-312, 1987.

FERREIRA, M.M.; FERNANDES, B.; CURI, N. Mineralogia da fração argila e estrutura de Latossolos da região sudeste do Brasil. Revista Brasileira de Ciência do Solo, Viçosa, v.23, p.507-514, 1999.

GUimarÃES, C.M.; STONE, L.F.; MOREIRA, A.A.J. Compactação do solo na cultura do feijoeiro. II: efeito sobre o desenvolvimento radicular e da parte aérea. Revista Brasileira de Engenharia Agrícola e Ambiental, Campina Grande, v.6, p.213-218, 2002.

HAMBLIM, A.P. The influence of soil structure on water movement, crop root growth and water uptake. Advances in Agronomy, New York, v.38, p.95-158, 1985.

KLUTE, A. Water retention: laboratory methods. In: KLUTE, A. Methods of soil analysis. 2.ed. Madison: American Society of Agronomy, 1986. V.1, p.635-662. 
KOOISTRA, M.J. et al. Root-soil contact of maize, as measured by a thin-section technique. II. Effects of soil compaction. Plant and Soil, Dorbrecht, v.139, p.119-129, 1992.

KOPI, A.J.; DOUGLAS, J.T. A rapid inexpensive and quantitative procedure for assessing soil structure with respect to cropping. Soil Use and Management, v.7, p.52-56, 1991.

LETEY, J. Relationship between soil physical properties and crop production. Advances in Soil Science, New York, v.1, p.277-294, 1985.

MEROTTO, A.J.; MUNDSTOCK, C.M. Wheat root growth as affected by soil strength. Revista Brasileira de Ciência do Solo, Viçosa, v.23, p.197-202, 1999.

MORAES, M.H.; BENEZ, S.H.; LIBARDI, P.L. Influência de camadas compactadas de subsuperfície no desenvolvimento do sistema radicular de plantas de soja (Glycine max (L) Merrill). Científica, São Paulo, v.19, p.195-206, 1991

MORAES, M.H. et al. Efeito da compactação subsuperficial do solo no desenvolvimento inicial e no estado nutricional de plantas de feijoeiro (Phaseolus vulgaris L.). Engenharia Agrícola, Jaboticabal, v.18, p.35-43, 1998.

RAIJ, B. van et al. Recomendação de adubação e calagem para o estado de São Paulo. 2.ed. Campinas : INSTITUTO AGRONÔMICO \& FUNDAÇÃO IAC, 1996. 285p. (Boletim Técnico, 100).

RAIJ, B. van et al. Análise química do solo para fins de fertilidade. Campinas : FUNDAÇÃO CARGILL, 1987. 170p.
ROSOLEM, C.A. et al. Crescimento radicular de plântulas de milho afetado pela resistência do solo à penetração. Pesquisa Agropecuária Brasileira, Brasília, v.34, p.821828, 1999.

SAS INSTITUTE. SAS/STAT user's guide. version 6. Cary, 1995. 1686p.

STONE, L.F.; GUIMARÃES, C.M.; MOREIRA, A.A.J. Compactação do solo na cultura do feijoeiro. I: efeitos nas propriedades físico-hídricas do solo. Revista Brasileira de Engenharia Agrícola e Ambiental, Campina Grande, v.6, p.207-212, 2002.

TAYLOR, H.; GARDNER, H. Penetration of cotton seedling taproots as influenced by bulk density, moisture content, and strength. Soil Science, Baltimore, v.963, p.153-156, 1963.

TIMLIM, D.J.; AHUJA, L.R.; ANKENY, M.D Comparison of three field methods to characterize apparent macropore conductivity. Soil Science Society of America Journal, Madison, v.58, p.278284,1994

TORMENA, C.A.; SILVA, A.P. da; LIBARDI, P.L. Caracterização do intervalo hídrico ótimo de um Latossolo Roxo sob plantio direto. Revista Brasileira de Ciência do Solo, Viçosa, v.22, p.573-581, 1998

VIEIRA, M.J.; MUZILLI, O. Características físicas de um Latossolo Vermelho Escuro sob diferentes sistemas de manejo. Pesquisa Agropecuária Brasileira, Brasília, v.19, p.873882, 1984. 